\title{
HAMILTON-JACOBI-BELLMAN EQUATIONS FOR THE OPTIMAL CONTROL OF A STATE EQUATION WITH MEMORY
}

\author{
${\text { Guillaume } \text { Carlier }^{1} \text { and Rabah Tahraoui }}^{1}$
}

Abstract. This article is devoted to the optimal control of state equations with memory of the form:

$$
\begin{array}{r}
\dot{x}(t)=F\left(x(t), u(t), \int_{0}^{+\infty} A(s) x(t-s) \mathrm{d} s\right), t>0, \\
\text { with initial conditions } x(0)=x, x(-s)=z(s), s>0 .
\end{array}
$$

Denoting by $y_{x, z, u}$ the solution of the previous Cauchy problem and:

$$
v(x, z):=\inf _{u \in V}\left\{\int_{0}^{+\infty} \mathrm{e}^{-\lambda s} L\left(y_{x, z, u}(s), u(s)\right) \mathrm{d} s\right\}
$$

where $V$ is a class of admissible controls, we prove that $v$ is the only viscosity solution of an HamiltonJacobi-Bellman equation of the form:

$$
\lambda v(x, z)+H\left(x, z, \nabla_{x} v(x, z)\right)+\left\langle D_{z} v(x, z), \dot{z}\right\rangle=0
$$

in the sense of the theory of viscosity solutions in infinite-dimensions of Crandall and Lions.

Mathematics Subject Classification. 49L20, 49L25.

Received March 21, 2008. Revised March 26, 2009.

Published online July 31, 2009.

\section{INTRODUCTION}

The optimal control of dynamics with memory is an issue that naturally arises in many different applied settings both in engineering and decision sciences. It is typically the case when studying the optimal performances of a system in which the response to a given input occurs not instantaneously but only after a certain elapse of time. To cite some recent related contributions, in a stochastic framework, we refer to Elsanosi et al. [14], for applications to mathematical finance, and to Gozzi and Marinelli [18] for applications to advertising modelling.

\footnotetext{
Keywords and phrases. Dynamic programming, state equations with memory, viscosity solutions, Hamilton-Jacobi-Bellman equations in infinite dimensions.

1 Université Paris Dauphine, CEREMADE, Pl. de Lattre de Tassigny, 75775 Paris Cedex 16, France.

carlier@ceremade.dauphine.fr, tahraoui@ceremade.dauphine.fr
} 
In the deterministic case, we refer to Boucekkine et al. [5] for a generalization of Ramsey's economic growth model with memory effects and, in the field of biosciences modelling, we refer to the survey of Baker et al. [1].

Motivated by economic problems, Fabbri et al. [16], Faggian and Gozzi [17] studied specific models using the dynamic programming approach. In these papers, the delay appears also in the control variable (a case we do not treat here) but the state equation is linear. Fabbri in [15] also considered the optimal control of a linear delay equation, proved that the value function is a viscosity solution of an HJB equation in infinite dimensions and gave a verification theorem. Compared with the recent literature mentioned previously, the present paper addresses, in a rather self-contained way, the dynamic principle approach and its viscosity counterpart for a class of control problems with memory and a nonlinear state equation. Using a notion of viscosity solution that slightly differs from the original one of Crandall and Lions (but is, we believe, easier to handle in the framework of equations with memory) we prove a comparison result and thus fully characterize the value function for such problems.

The aim of the present article is to study, by dynamic programming arguments, the optimal control of (deterministic) state equations with memory. For the sake of simplicity, we will restrict the analysis to (finitedimensional) dynamics of the form:

$$
\dot{x}(t)=F\left(x(t), u(t), \int_{0}^{+\infty} A(s) x(t-s) \mathrm{d} s\right), t>0,
$$

with initial conditions $x(0)=x$ and $x(-s)=z(s), s>0$. We will also focus on the discounted infinite horizon problem:

$$
v(x, z):=\inf _{u \in V}\left\{\int_{0}^{+\infty} \mathrm{e}^{-\lambda s} L\left(y_{x, z, u}(s), u(s)\right) \mathrm{d} s\right\}
$$

where $V$ is some admissible class of controls. Of course, there are other forms of memory effects than the one we treat here: systems with lags or with deviating arguments for instance (see for instance $[7,21,22]$ and the references therein).

As is obvious from (1.1), the value function depends not only on the current state of the system $x$ but also on the whole past of the trajectory i.e. $z$ (note that we have not required $z(0)=x$ in $(1.1)$ ). Hence the state space for problem (1.1) is infinite-dimensional (we refer to the classical books [2,19] for a general theory and more examples regarding the control of infinite-dimensional systems). Note that if we had imposed an additional continuity condition ensuring $x=z(0)$, then the value function would have been a function of the past $z$ only. There are several reasons why we have not adopted this point of view and have preferred to write everywhere $x$ and $z$ as if they were independent variables. The main one, is that it enables to understand the tight connections between the control problem (1.1) and the Hamilton-Jacobi-Bellman equation:

$$
\lambda v(x, z)+H\left(x, z, \nabla_{x} v(x, z)\right)+\left\langle D_{z} v(x, z), \dot{z}\right\rangle=0 \text { if } z(0)=x .
$$

The previous equation presents several difficulties. The first one is of course its infinite-dimensional nature. The second one comes from the presence of the time derivative of $z, \dot{z}$ in the equation and the third one from the restriction $x=z(0)$. In a series of articles [8-13], Crandall and Lions developed a general theory of viscosity solutions in infinite dimensions. This theory is of course of particular interest for the optimal control of infinitedimensional systems. In such problems, the Hamilton-Jacobi equation frequently contains an unbounded linear term (as in (1.2)) and in [11-13], Crandall and Lions showed how to overcome this additional difficulty. The main contribution of the present paper is to show, in a rather simple and self-contained way, how the theory of viscosity solutions in infinite dimensions of Crandall and Lions can be applied to fully characterize the value function (1.1) as the unique solution of (1.2). For the sake of simplicity, we will work in a Hilbertian framework i.e. in the state space $E:=\mathbb{R}^{d} \times L^{2}\left(\mathbb{R}_{+}, \mathbb{R}^{d}\right)$ and defining:

$$
E_{0}:=\left\{(z(0), z), z \in H^{1}\left(\mathbb{R}_{+}, \mathbb{R}^{d}\right)\right\}
$$


$v$ will be said to be a viscosity subsolution of (1.2) if for every $\left(x_{0}, z_{0}\right) \in \mathbb{R}^{d} \times L^{2}$ and every $\phi \in C^{1}\left(\mathbb{R}^{d} \times L^{2}, \mathbb{R}\right)$ such that $v-\phi$ has a local maximum (in the sense of the strong topology of $\left.\mathbb{R}^{d} \times L^{2}\right)$ at $\left(x_{0}, z_{0}\right)$, one has:

$$
\lambda v\left(x_{0}, z_{0}\right)+H\left(x_{0}, z_{0}, \nabla_{x} \phi\left(x_{0}, z_{0}\right)\right)+\liminf _{(x, z) \in E_{0} \rightarrow\left(x_{0}, z_{0}\right)}\left\langle D_{z} \phi(x, z), \dot{z}\right\rangle \leq 0 .
$$

Supersolutions of (1.2) are defined in a similar way. Now, a convenient way to study (1.2) is to rewrite it as an Hamilton-Jacobi equation with an unbounded linear term as in Crandall and Lions [11-13]. Namely, defining $\alpha=(x, z)$, the equation reads as:

$$
\lambda v(\alpha)+H\left(\alpha, \nabla_{x} v(\alpha)\right)-x \cdot \nabla_{x} v(\alpha)+\left\langle T^{*}(\alpha), D v(\alpha)\right\rangle=0, \alpha \in D\left(T^{*}\right),
$$

where $T$ is the linear unbounded operator on $E$ with domain $D(T)=\mathbb{R}^{d} \times H^{1}$ defined by

$$
T(y, w):=(y-w(0),-\dot{w}), \forall(y, w) \in D(T) .
$$

So that its adjoint, $T^{*}$ has domain $D\left(T^{*}\right)=E_{0}$ and is given by

$$
T^{*}(x, z):=(z(0), \dot{z})=(x, \dot{z}), \forall(x, z) \in D\left(T^{*}\right)=E_{0} .
$$

Section 2 is devoted to some preliminaries on the Cauchy problem and continuity properties of the value function. Section 3 concerns the dynamic programming principle. In Section 4, we identify the Hamilton-JacobiBellman equation of the problem and establish that the value function is a viscosity solution of this equation. In Section 5, we prove a comparison result. Finally, in Section 6, we end the paper by some concluding remarks.

\section{Assumptions AND PRELiminaries}

\subsection{On the Cauchy problem}

Let $K$ be a compact metric space, we define the set of admissible controls $V$ as the set of measurable functions on $(0,+\infty)$ with values in $K$. For $z \in L^{2}:=L^{2}\left((0,+\infty), \mathbb{R}^{d}\right), x \in \mathbb{R}^{d}$ and $u \in V$ an admissible control, we consider the following controlled equation

$$
\dot{x}(t)=F\left(x(t), u(t), \int_{0}^{+\infty} A(s) x(t-s) \mathrm{d} s\right), t>0,
$$

together with the boundary conditions:

$$
x(0)=x, x(-s)=z(s), s>0 .
$$

In the paper, $d$ and $k$ are given positive integers and we will always assume the following on the data $A$ and $F$ :

- (H1) $F \in C^{0}\left(\mathbb{R}^{d} \times K \times \mathbb{R}^{k}, \mathbb{R}^{d}\right)$ and there exists a constant $C_{1} \geq 0$ such that:

$$
|F(x, u, \alpha)-F(y, u, \beta)| \leq C_{1}(|x-y|+|\alpha-\beta|),
$$

for every $(x, y, \alpha, \beta, u) \in \mathbb{R}^{d} \times \mathbb{R}^{d} \times \mathbb{R}^{k} \times \mathbb{R}^{k} \times V$;

- (H2) $A \in L^{2}\left((0,+\infty), M_{k \times d}\right) \cap L^{1}\left((0,+\infty), M_{k \times d}\right)$ ( $M_{k \times d}$ standing for the space of real matrices with $k$ rows and $d$ columns).

In the sequel, we shall sometimes use a stronger assumption than (H2). Namely:

(H2') $A \in H^{1}\left((0,+\infty), M_{k \times d}\right) \cap L^{1}\left((0,+\infty), M_{k \times d}\right)$.

At this point, let us mention that we will always identify $L^{2}$ with its dual and therefore we won't identify $H^{1}$ and $\left(H^{1}\right)^{\prime}$. 
Before studying the optimal control of equations with memory of type (2.1), let us establish the existence, uniqueness and continuous dependence with respect to initial conditions for the Cauchy problem (2.1)-(2.2). The results of this section (Props. 2.1 and 2.2) are fairly standard but we give proofs for the sake of completeness and to keep the present paper self-contained. Conditions (H1) and (H2) of course ensure existence and uniqueness of a solution to the Cauchy problem (2.1)-(2.2):

Proposition 2.1. Assume that (H1) and (H2) hold. For every $(x, z, u) \in \mathbb{R}^{d} \times L^{2} \times V$, the Cauchy problem (2.1)-(2.2) admits a unique solution.

Proof. For $\theta>0$, define

$$
E_{\theta}:=\left\{y \in C^{0}\left(\mathbb{R}_{+}, \mathbb{R}^{d}\right), \sup _{t \geq 0} \mathrm{e}^{-\theta t}|y(t)|<+\infty\right\}
$$

and equip $E_{\theta}$ with the norm:

$$
\|y\|_{\theta}:=\sup _{t \geq 0} \mathrm{e}^{-\theta t}|y(t)| .
$$

Of course, $\left(E_{\theta},\|\cdot\|_{\theta}\right)$ is a Banach space. For $y \in E_{\theta}$, let us define:

$$
T y(t):=x+\int_{0}^{t} F\left(y(s), u(s), G_{y}(s)\right) \mathrm{d} s, \forall t \geq 0
$$

where

$$
G_{y}(s):=\int_{0}^{s} A(\tau) y(s-\tau) \mathrm{d} \tau+\int_{s}^{+\infty} A(\tau) z(\tau-s) \mathrm{d} \tau .
$$

Until the end of the proof, $C$ will denote a positive constant (only depending on $F$ and $A$ ) which may vary from one line to another. Let $y \in E_{\theta}$, with assumption $(\mathbf{H} 1)$ on $F$, we first get:

$$
|T y(t)| \leq|x|+C\left(t+\frac{\mathrm{e}^{\theta t}}{\theta}\|y\|_{\theta}+\int_{0}^{t}\left|G_{y}(s)\right| \mathrm{d} s\right) .
$$

Now, with (H2) we also have

$$
\begin{aligned}
\left|G_{y}(s)\right| & \leq\left(\int_{0}^{s}\left|A(\tau)\|y(s-\tau) \mid \mathrm{d} \tau+\| A\left\|_{L^{2}}\right\| z \|_{L^{2}}\right)\right. \\
& \leq\|A\|_{L^{2}}\left(\|y\|_{\theta}\left(\int_{0}^{s} \mathrm{e}^{2 \theta(s-\tau)} \mathrm{d} \tau\right)^{1 / 2}+\|z\|_{L^{2}}\right) \\
& \leq C\left(1+\frac{\mathrm{e}^{\theta s}\|y\|_{\theta}}{\sqrt{2 \theta}}\right) .
\end{aligned}
$$

Together with (2.4), we then have

$$
|T y(t)| \mathrm{e}^{-\theta t} \leq|x| \mathrm{e}^{-\theta t}+C\left(t \mathrm{e}^{-\theta t}+\|y\|_{\theta}\left(\frac{1}{\theta}+\frac{1}{\sqrt{2} \theta^{3 / 2}}\right)\right)
$$

which proves that $T\left(E_{\theta}\right) \subset E_{\theta}$. For $y_{1}$ and $y_{2}$ in $E_{\theta}$ and $t \geq 0$, on the one hand, we have:

$$
\left|T y_{1}(t)-T y_{2}(t)\right| \leq C\left(\frac{\mathrm{e}^{\theta t}}{\theta}\left\|y_{1}-y_{2}\right\|_{\theta}+\int_{0}^{t}\left|G_{y_{1}}(s)-G_{y_{2}}(s)\right| \mathrm{d} s\right)
$$

on the other hand:

$$
\left|G_{y_{1}}(s)-G_{y_{2}}(s)\right| \leq \frac{C \mathrm{e}^{\theta s}}{\sqrt{2 \theta}}\left\|y_{1}-y_{2}\right\|_{\theta}
$$


so that:

$$
\left\|T y_{1}-T y_{2}\right\|_{\theta} \leq C\left\|y_{1}-y_{2}\right\|_{\theta}\left(\frac{1}{\theta}+\frac{1}{\sqrt{2} \theta^{3 / 2}}\right) .
$$

For $\theta$ large enough $\left(\theta \geq 2 C+1\right.$, say), $T$ is a contraction of $E_{\theta}$ hence admits a unique fixed-point. This clearly proves the desired result.

From now on, for every $(x, z, u) \in \mathbb{R}^{d} \times L^{2} \times V$, we denote by $y_{x, z, u}$ the solution of the Cauchy problem (2.1)-(2.2). The continuous dependence with respect to $(x, z)$ of trajectories of $(2.1)-(2.2)$ is given by:

Proposition 2.2. Assume that (H1) and (H2) hold. Let $u \in V,\left(x_{0}, z_{0}\right)$ and $(x, z)$ be in $\mathbb{R}^{d} \times L^{2}$ and define $y_{0}:=y_{x_{0}, z_{0}, u}, y:=y_{x, z, u}$, then we have

$$
\left|y(t)-y_{0}(t)\right| \leq C \mathrm{e}^{\theta t}\left(\left|x-x_{0}\right|+\left\|z-z_{0}\right\|_{L^{2}}\right), \forall t \geq 0
$$

for some constants $C$ and $\theta$ depending only on $F$ and $A$.

Proof. In this proof, $C$ will denote a positive constant that only depends on $F$ and $A$ but which may vary from one line to another. Defining for $s \geq 0$

$$
\begin{aligned}
\beta(s) & :=\int_{0}^{s} A(s-\tau) y(\tau) \mathrm{d} \tau+\int_{0}^{+\infty} A(s+\tau) z(\tau) \mathrm{d} \tau, \\
\beta_{0}(s) & :=\int_{0}^{s} A(s-\tau) y_{0}(\tau) \mathrm{d} \tau+\int_{0}^{+\infty} A(s+\tau) z_{0}(\tau) \mathrm{d} \tau, \\
\gamma(s) & :=\sup _{[0, s]}\left|y-y_{0}\right|, \Gamma(s):=\int_{0}^{s} \gamma,
\end{aligned}
$$

we first have:

Since we also have

$$
\left|y(s)-y_{0}(s)\right| \leq\left|x-x_{0}\right|+C\left(\int_{0}^{s}\left(\left|y-y_{0}\right|+\left|\beta-\beta_{0}\right|\right)\right) .
$$

for $t \geq 0$, we then get:

$$
\left|\beta(\tau)-\beta_{0}(\tau)\right| \leq C\left(\gamma(\tau)\|A\|_{L^{1}}+\|A\|_{L^{2}}\left\|z-z_{0}\right\|_{L^{2}}\right)
$$

$$
\Gamma^{\prime}(t) \leq\left|x-x_{0}\right|+C\left(\Gamma(t)+\left\|z-z_{0}\right\|_{L^{2}} t\right)
$$

which, together with Gronwall's Lemma gives the desired result.

Remark 2.1. Let us remark that when one further assumes that (H'2) holds (i.e. A is further assumed to be $\left.H^{1}\right)$, then the estimate of Proposition 2.2 also holds true when one replaces $\left\|z-z_{0}\right\|_{L^{2}}$ by $\left\|z-z_{0}\right\|_{\left(H^{1}\right)^{\prime}}$.

\subsection{The optimal control problem}

For $(x, z) \in \mathbb{R}^{d} \times L^{2}$, we consider the optimal control problem

$$
v(x, z):=\inf _{u \in V} \int_{0}^{+\infty} \mathrm{e}^{-\lambda s} L\left(y_{x, z, u}(s), u(s)\right) \mathrm{d} s,
$$

where (H3): $\lambda>0$ and $L: \mathbb{R}^{d} \times K \rightarrow \mathbb{R}$ is assumed to be bounded, continuous and to satisfy

$$
|L(x, u)-L(y, u)| \leq C_{2}|x-y|, \forall(x, y, u) \in \mathbb{R}^{d} \times \mathbb{R}^{d} \times K
$$

for some $C_{2} \geq 0$. Throughout the paper, we will assume that (H1), (H2) and (H3) hold. 


\subsection{Continuity properties of the value function}

As a consequence of Proposition 2.2, we deduce that $v$ is bounded and uniformly continuous on $\mathbb{R}^{d} \times L^{2}$, which we denote $v \in \mathrm{BUC}\left(\mathbb{R}^{\mathrm{d}} \times \mathrm{L}^{2}, \mathbb{R}\right)$. More precisely, adapting classical arguments (see e.g. Barles [4]) to our context, we have:

Proposition 2.3. Assume that (H1), (H2) and (H3) hold, then $v \in \mathrm{BUC}\left(\mathbb{R}^{\mathrm{d}} \times \mathrm{L}^{2}, \mathbb{R}\right)$ and more precisely, defining $\theta$ as in Proposition 2.2, one has:

(1) $v$ is Lipschitz continuous on $\mathbb{R}^{d} \times L^{2}$ if $\lambda>\theta$;

(2) $v \in C^{0, \lambda / \theta}\left(\mathbb{R}^{d} \times L^{2}, \mathbb{R}\right)$ if $\lambda<\theta$;

(3) $v \in C^{0, \alpha}\left(\mathbb{R}^{d} \times L^{2}, \mathbb{R}\right)$ for every $\alpha \in(0,1)$ if $\lambda=\theta$.

Proof. Let us define

$$
\delta:=\left|x-x_{0}\right|+\left\|z-z_{0}\right\|_{L^{2}} .
$$

Let $\varepsilon>0$ and $u_{\varepsilon}$ be such that

$$
\int_{0}^{+\infty} \mathrm{e}^{-\lambda s} L\left(y_{x, z, u_{\varepsilon}}(s), u_{\varepsilon}(s)\right) \mathrm{d} s \leq v(x, z)+\varepsilon
$$

setting $y^{\varepsilon}:=y_{x, z, u_{\varepsilon}}, y_{0}^{\varepsilon}:=y_{x_{0}, z_{0}, u_{\varepsilon}}$ we then have:

$$
v\left(x_{0}, z_{0}\right)-v(x, z) \leq \int_{0}^{+\infty} \mathrm{e}^{-\lambda s}\left(L\left(y_{0}^{\varepsilon}(s), u_{\varepsilon}(s)\right)-L\left(y^{\varepsilon}(s), u_{\varepsilon}(s)\right)\right) \mathrm{d} s+\varepsilon .
$$

Using Proposition 2.2 and assumption (H3) on $L$, we then get, for some $C \geq 0$ and all $T \geq 0$ :

$$
v\left(x_{0}, z_{0}\right)-v(x, z) \leq C\left(\int_{0}^{T} \delta \mathrm{e}^{(\theta-\lambda) s} \mathrm{~d} s+\mathrm{e}^{-\lambda T}\right) .
$$

If $\lambda>\theta$, we then have:

which proves the first claim.

$$
v\left(x_{0}, z_{0}\right)-v(x, z) \leq \frac{C \delta}{\lambda-\theta}
$$

If $\lambda<\theta$ and if $\delta<1$ (which may be assumed to prove that $v$ is Hölder) taking $\mathrm{e}^{-\lambda T}:=\delta^{\lambda / \theta}$ in (2.7) then yields

$$
v\left(x_{0}, z_{0}\right)-v(x, z) \leq C\left(1+\frac{1}{\theta-\lambda}\right) \delta^{\lambda / \theta}
$$

which proves the second claim.

Finally, if $\lambda=\theta$ (and again assuming $\delta<1$ ), taking $\mathrm{e}^{-\lambda T}=\delta$ in (2.7) yields:

$$
v\left(x_{0}, z_{0}\right)-v(x, z) \leq C\left(\frac{-\delta \log (\delta)}{\lambda}+\delta\right)
$$

which proves the last claim.

Remark 2.2. Again, if $A$ is further assumed to be $H^{1}$ (i.e. when (H'2) holds) then the uniform continuity of $v$ also holds true for the norm $(x, z) \mapsto|x|+\|z\|_{\left(H^{1}\right)^{\prime}}$ i.e. when in the previous proof $\delta$ is replaced by $\delta:=\left|x-x_{0}\right|+\left\|z-z_{0}\right\|_{\left(H^{1}\right)^{\prime}}$. This fact will be useful later on when proving the comparison result. 
In the sequel, we shall denote by $C^{0}\left(\mathbb{R}^{d} \times \mathrm{L}_{\mathrm{w}}^{2}, \mathbb{R}\right)$ the class of real-valued functions defined on $\mathbb{R}^{d} \times L^{2}$ which are sequentially continuous for the weak topology of $\mathbb{R}^{d} \times L^{2}$, we then have the following:

Proposition 2.4. Assume that (H1), (H2) and (H3) hold, then $v \in C^{0}\left(\mathbb{R}^{d} \times \mathrm{L}_{\mathrm{w}}^{2}, \mathbb{R}\right)$.

Proof. Let $\left(\alpha_{n}\right)_{n}:=\left(z_{n}, x_{n}\right)_{n}$ be a weakly convergent sequence in $\mathbb{R}^{d} \times L^{2}$ and let us denote by $\alpha:=(x, z) \in$ $\mathbb{R}^{d} \times L^{2}$ its weak limit. Let $u \in V$ be some admissible control and simply denote $y_{n}:=y_{\alpha_{n}, u}$ and $y:=$ $y_{\alpha, u}$ the trajectories of (2.1) associated respectively to the initial conditions $\alpha_{n}$ and $\alpha$. If we prove that $y_{n}$ converges uniformly on compact subsets to $y$ as $n$ tends to $+\infty$ then the desired result will easily follow from our assumptions on $L$. Let us define

$$
\delta_{n}(t):=\int_{0}^{+\infty} A(t+s) z_{n}(s) \mathrm{d} s, \delta(t):=\int_{0}^{+\infty} A(t+s) z(s) \mathrm{d} s .
$$

Since $A \in L^{2}$ by (H2), we have:

$$
\left|\delta_{n}(t)\right| \leq\|A\|_{L^{2}}\left\|z_{n}\right\|_{L^{2}} \leq C .
$$

Thanks to (H2) again, $\delta_{n}$ converges pointwise to $\delta$. Rewriting the state equation as:

$$
\begin{gathered}
\dot{y}(t)=F\left(y(t), u(t), \delta(t)+\int_{0}^{t} A(t-s) y(s) \mathrm{d} s\right), \\
\dot{y}_{n}(t)=F\left(y_{n}(t), u(t), \delta_{n}(t)+\int_{0}^{t} A(t-s) y_{n}(s) \mathrm{d} s\right)
\end{gathered}
$$

we get:

$$
\left|\dot{y}_{n}-\dot{y}\right|(t) \leq C\left(\left|y_{n}-y\right|(t)+\left|\delta_{n}-\delta\right|(t)+\int_{0}^{t}\left|A(t-s)\left(y_{n}-y\right)(s)\right| \mathrm{d} s\right)
$$

(where again in this proof $C$ denotes a nonnegative constant depending only on $F$ and $A$ but possibly changing from one line to another). Defining

$$
\gamma_{n}(t):=\sup _{[0, t]}\left|y_{n}-y\right|, \Gamma_{n}(t):=\int_{0}^{t} \gamma_{n},
$$

inequality (2.8) yields for all $s \in[0, t]$ :

$$
\left|\dot{y}_{n}-\dot{y}\right|(s) \leq C\left(\left|y_{n}-y\right|(s)+\left|\delta_{n}-\delta\right|(s)+\|A\|_{L^{1}(0, t)} \gamma_{n}(s)\right) .
$$

Integrating the previous yields:

$$
\left|y_{n}-y\right|(s) \leq\left|x_{n}-x\right|+C\left(\Gamma_{n}(t)+\int_{0}^{t}\left|\delta_{n}-\delta\right|\right), \forall s \in[0, t] .
$$

Hence

$$
\gamma_{n}(t)=\dot{\Gamma}_{n}(t) \leq\left|x_{n}-x\right|+C\left(\Gamma_{n}(t)+\int_{0}^{t}\left|\delta_{n}-\delta\right|\right) .
$$

On the one hand, dominated convergence implies that

$$
\lim _{n} \int_{0}^{t}\left|\delta_{n}-\delta\right|=0, \forall t \in[0,+\infty)
$$

on the other hand, (2.9) and Gronwall's Lemma imply that $\Gamma_{n}(t)$ tends to 0 as $n$ tends to $+\infty$. With (2.9), this proves that $\gamma_{n}(t)$ tends to 0 as $n$ tends to $+\infty$ and the desired result follows. Let us remark that, with (2.8), this of course also implies that $\left(y_{n}\right)_{n}$ converges to $y$ in $W_{\text {loc }}^{1, \infty}\left(\mathbb{R}_{+}, \mathbb{R}^{d}\right)$. 


\section{DyNAMIC PROGRAMMING PRINCIPLE}

Our aim now is to prove that the value function $v$ obeys the following dynamic programming principle:

Proposition 3.1. Let $(x, z) \in \mathbb{R}^{d} \times L^{2}$ and $t \geq 0$, we then have:

$$
v(x, z)=\inf _{u \in V}\left\{\int_{0}^{t} \mathrm{e}^{-\lambda s} L\left(y_{x, z, u}(s), u(s)\right) \mathrm{d} s+\mathrm{e}^{-\lambda t} v\left(y_{x, z, u}(t), y_{x, z, u}(t-.)\right)\right\}
$$

$\left(y_{x, z, u}(t-).(s):=y_{x, z, u}(t-s)\right.$ for all $\left.s>0\right)$.

Proof. Let $\varepsilon>0$ and $u_{\varepsilon} \in V$ be such that

$$
\int_{0}^{+\infty} \mathrm{e}^{-\lambda s} L\left(y_{x, z, u_{\varepsilon}}(s), u_{\varepsilon}(s)\right) \mathrm{d} s \leq v(x, z)+\varepsilon
$$

we then have

$$
v(x, z)+\varepsilon \geq \int_{0}^{t} \mathrm{e}^{-\lambda s} L\left(y_{x, z, u_{\varepsilon}}(s), u_{\varepsilon}(s)\right) \mathrm{d} s+\mathrm{e}^{-\lambda t} \int_{0}^{+\infty} \mathrm{e}^{-\lambda \tau} L\left(y_{x, z, u_{\varepsilon}}(t+\tau), u_{\varepsilon}(t+\tau)\right) \mathrm{d} \tau .
$$

Using the fact that $y_{x, z, u_{\varepsilon}}(t+$.$) is the trajectory associated to the initial conditions \left(y_{x, z, u_{\varepsilon}}(t), y_{x, z, u_{\varepsilon}}(t-).\right)$ and the control $u_{\varepsilon}(t+$.$) , we deduce:$

$$
\begin{aligned}
v(x, z)+\varepsilon & \geq \int_{0}^{t} \mathrm{e}^{-\lambda s} L\left(y_{x, z, u_{\varepsilon}}(s), u_{\varepsilon}(s)\right) \mathrm{d} s+\mathrm{e}^{-\lambda t} v\left(y_{x, z, u_{\varepsilon}}(t), y_{x, z, u_{\varepsilon}}(t-.)\right) \\
& \geq \inf _{u \in V}\left\{\int_{0}^{t} \mathrm{e}^{-\lambda s} L\left(y_{x, z, u}(s), u(s)\right) \mathrm{d} s+\mathrm{e}^{-\lambda t} v\left(y_{x, z, u}(t), y_{x, z, u}(t-.)\right)\right\} .
\end{aligned}
$$

To prove the converse inequality, let $u \in V$,

$$
x_{t}:=y_{x, z, u}(t), z_{t}:=y_{x, z, u}(t-.),
$$

$\varepsilon>0$ and $\omega_{\varepsilon} \in V$ be such that:

$$
\int_{0}^{+\infty} \mathrm{e}^{-\lambda s} L\left(y_{x_{t}, z_{t}, \omega_{\varepsilon}}(s), \omega_{\varepsilon}(s)\right) \mathrm{d} s \leq v\left(x_{t}, z_{t}\right)+\varepsilon
$$

defining

we have

$$
u_{\varepsilon}(s):= \begin{cases}u(s) & \text { if } s \in[0, t] \\ \omega_{\varepsilon}(s-t) & \text { if } s>t\end{cases}
$$

hence

$$
y_{x, z, u_{\varepsilon}}(s):= \begin{cases}y_{x, z, u}(s) & \text { if } s \in[0, t] \\ y_{x_{t}, z_{t}, \omega_{\varepsilon}}(s-t) & \text { if } s>t\end{cases}
$$

$$
\begin{aligned}
v(x, z) & \leq \int_{0}^{t} \mathrm{e}^{-\lambda s} L\left(y_{x, z, u}(s), u(s)\right) \mathrm{d} s+\int_{t}^{+\infty} \mathrm{e}^{-\lambda s} L\left(y_{x_{t}, z_{t}, \omega_{\varepsilon}}(s-t), \omega_{\varepsilon}(s-t)\right) \mathrm{d} s \\
& \leq \int_{0}^{t} \mathrm{e}^{-\lambda s} L\left(y_{x, z, u}(s), u(s)\right) \mathrm{d} s+\mathrm{e}^{-\lambda t}\left(v\left(x_{t}, z_{t}\right)+\varepsilon\right)
\end{aligned}
$$

since $u$ and $\varepsilon>0$ are arbitrary, we get the desired result. 


\section{Viscosity solutions And HAMILton-Jacobi-Bellman EQUATIONS}

\subsection{Preliminaries}

Let us define

$$
E_{0}:=\left\{(z(0), z), z \in H^{1}\left((0,+\infty), \mathbb{R}^{d}\right)\right\}
$$

and remark that $E_{0}$ is a dense subspace of our initial state space $E:=\mathbb{R}^{d} \times L^{2}$. With the uniform continuity of $v$ on $\mathbb{R}^{d} \times L^{2}$, this implies that $v$ is fully determined by its restriction to $E_{0}$. In fact, we will derive from the dynamic programming principle a PDE satisfied by $v$ (a priori only on $E_{0}$ ) and by a comparison result we will see that in fact this equation (satisfied in some appropriate viscosity sense) fully characterizes the value function $v$. Let us define

$$
\mathcal{F}(x, z, u):=F\left(x, u, \int_{0}^{+\infty} A(s) z(s) \mathrm{d} s\right), \forall(x, z, u) \in \mathbb{R}^{d} \times L^{2} \times K .
$$

Before going further, we need the following classical lemma (see for instance [6], Lem. IV.4):

Lemma 4.1. Let $\delta>0$ and $z \in L^{2}\left((-\delta,+\infty), \mathbb{R}^{d}\right)$ for $t \in[0, \delta]$, define $z_{t}(s):=z(s-t)$ for $s \geq 0$, then $z_{t}$ converges to $z$ in $L^{2}\left(\mathbb{R}_{+}, \mathbb{R}^{d}\right)$ as $t$ goes to $0^{+}$.

We will also need the following

Lemma 4.2. Let $(x, z) \in E_{0}, u \in V$ and for $t>0$ define:

$$
x_{t}:=y_{x, z, u}(t), z_{t}(\tau):=y_{x, z, u}(t-\tau), \forall \tau>0,
$$

then $t \mapsto\left(x_{t}, z_{t}\right)$ is locally Lipschitz in $t$ (uniformly in the control $u \in V$ ) for the $\mathbb{R}^{d} \times L^{2}$ norm. Moreover, for all $t \geq 0$

$$
\lim _{s \rightarrow 0^{+}} \frac{z_{t+s}-z_{t}}{s}=-\dot{z}_{t} \text { in } L^{2}
$$

and, for every $t$ which is a Lebesgue point of $t \mapsto \mathcal{F}\left(x_{t}, z_{t}, u(t)\right)$

$$
\lim _{s \rightarrow 0^{+}} \frac{x_{t+s}-x_{t}}{s}=\mathcal{F}\left(x_{t}, z_{t}, u(t)\right) .
$$

Proof. The lipschitzianity of $t \mapsto x_{t}$ and the proof of (4.4) are straightforward. To shorten notation, we define $y:=y_{x, z, u}$ and remark that for every $t \geq 0, y \in H^{1}\left((-\infty, t), \mathbb{R}^{d}\right)$ and that $y \in W_{\mathrm{loc}}^{1, \infty}\left((0,+\infty), \mathbb{R}^{d}\right)$ so that $(4.3)$ will imply the local lipschitzianity of $t \mapsto z_{t}$. To prove (4.3), let us introduce for $s>0$ and $\tau \geq 0$ :

$$
\Delta_{s}(\tau):=\frac{z_{t+s}(\tau)-z_{t}(\tau)}{s}+\dot{z}_{t}(\tau)
$$

which can be rewritten as:

$$
\begin{aligned}
\Delta_{s}(\tau) & =\frac{y(t+s-\tau)-y(t-\tau)}{s}-\dot{y}(t-\tau) \\
& =\frac{1}{s} \int_{0}^{s}(\dot{y}(t-\tau+\alpha)-\dot{y}(t-\tau)) \mathrm{d} \alpha
\end{aligned}
$$

Jensen's inequality first yields:

$$
\Delta_{s}(\tau)^{2} \leq \frac{1}{s} \int_{0}^{s}(\dot{y}(t-\tau+\alpha)-\dot{y}(t-\tau))^{2} \mathrm{~d} \alpha
$$


using Fubini's theorem, we then get:

$$
\left\|\Delta_{s}\right\|_{L^{2}}^{2} \leq \frac{1}{s} \int_{0}^{s}\left(\int_{\mathbb{R}_{+}}(\dot{y}(t-\tau+\alpha)-\dot{y}(t-\tau))^{2} \mathrm{~d} \tau\right) \mathrm{d} \alpha
$$

By the same arguments as in Lemma 4.1 and since $y \in H^{1}\left((-\infty, t), \mathbb{R}^{d}\right)$, for every $t \geq 0$, we deduce that for every $\varepsilon>0$, there exists $s_{\varepsilon}>0$ such that for all $\alpha \in\left[0, s_{\varepsilon}\right]$, one has:

$$
\left(\int_{\mathbb{R}_{+}}(\dot{y}(t-\tau+\alpha)-\dot{y}(t-\tau))^{2} \mathrm{~d} \tau\right) \leq \varepsilon
$$

which proves that $\Delta_{s}$ converges to 0 in $L^{2}$ as $s$ goes to 0 .

Remark 4.1. It follows from Lemma 4.2 that if $\psi \in C^{1}\left(\mathbb{R} \times \mathbb{R}^{d} \times L^{2}, \mathbb{R}\right)$ then the function $t \mapsto \psi\left(t, x_{t}, z_{t}\right)$ is locally Lipschitz in $t$ (in fact, uniformly in the control $u \in V$ ) and for all $t \geq 0$, one has:

$$
\psi\left(t, x_{t}, z_{t}\right)=\psi\left(0, x_{0}, z_{0}\right)+\int_{0}^{t}\left(\partial_{t} \psi\left(s, x_{s}, z_{s}\right)+\nabla_{x} \psi\left(s, x_{s}, z_{s}\right) \cdot \mathcal{F}\left(x_{s}, z_{s}, u(s)\right)-\left\langle D_{z} \psi\left(s, x_{s}, z_{s}\right), \dot{z}_{s}\right\rangle\right) \mathrm{d} s
$$

\subsection{Formal derivation of the equation}

To formally derive the Hamilton-Jacobi-Bellman equation of our problem, let us assume for a moment that $v$ is of class $C^{1}$ on $\mathbb{R}^{d} \times L^{2}$. Let $(x, z) \in E_{0}, u \in K$ be some admissible constant control, and set

$$
\left(x_{t}, z_{t}\right):=\left(y_{x, z, u}(t), y_{x, z, u}(t-.)\right),
$$

by the dynamic programming principle, we first have:

$$
v(x, z) \leq \int_{0}^{t} \mathrm{e}^{-\lambda s} L\left(x_{s}, u\right) \mathrm{d} s+\mathrm{e}^{-\lambda t} v\left(x_{t}, z_{t}\right)
$$

so that

$$
L(x, u)+\lim _{t \rightarrow 0^{+}} \frac{1}{t}\left(\mathrm{e}^{-\lambda t} v\left(x_{t}, z_{t}\right)-v(x, z)\right) \geq 0
$$

together with Lemma 4.2, this reads as

$$
L(x, u)+\nabla_{x} v(x, z) \cdot \mathcal{F}(x, z, u)-\lambda v(x, z)-\left\langle D_{z} v(x, z), \dot{z}\right\rangle \geq 0
$$

and since $u$ is arbitrary, this yields

$$
\lambda v(x, z)+\left\langle D_{z} v(x, z), \dot{z}\right\rangle+\sup _{u \in K}\left\{-L(x, u)-\nabla_{x} v(x, z) \cdot \mathcal{F}(x, z, u)\right\} \leq 0 .
$$

We then define the Hamiltonian:

$$
H(x, z, p):=\sup _{u \in K}\{-L(x, u)-p \cdot \mathcal{F}(x, z, u)\}, \forall(x, z, p) \in \mathbb{R}^{d} \times L^{2} \times \mathbb{R}^{d} .
$$

Let $u \in V$, and simply denote $\left(x_{t, u}, z_{t, u}\right):=\left(y_{x, z, u}(t), y_{x, z, u}(t-).\right)$. Using Remark 4.1 following Lemma 4.2 , with $\psi(t, x, z):=\mathrm{e}^{-\lambda t} v(x, z)$, we have:

$$
\begin{aligned}
\mathrm{e}^{-\lambda t} v\left(x_{t, u}, z_{t, u}\right)= & v(x, z)-\int_{0}^{t} \mathrm{e}^{-\lambda s} \lambda v\left(x_{s, u}, z_{s, u}\right) \mathrm{d} s+\int_{0}^{t} \mathrm{e}^{-\lambda s}\left(\nabla_{x} v\left(x_{s, u}, z_{s, u}\right) \cdot \mathcal{F}\left(x_{s, u}, z_{s, u}, u(s)\right)\right. \\
& \left.-\left\langle D_{z} v\left(x_{s, u}, z_{s, u}\right), \dot{z}_{s, u}\right\rangle\right) \mathrm{d} s
\end{aligned}
$$


so that the dynamic programming principle yields:

$$
\begin{aligned}
0= & \inf _{u \in V}\left\{\int _ { 0 } ^ { t } \mathrm { e } ^ { - \lambda s } \left(L\left(x_{s, u}, u(s)\right)-\lambda v\left(x_{s, u}, z_{s, u}\right)+\nabla_{x} v\left(x_{s, u}, z_{s, u}\right) \cdot \mathcal{F}\left(x_{s, u}, z_{s, u}, u(s)\right)\right.\right. \\
& \left.\left.-\left\langle D_{z} v\left(x_{s, u}, z_{s, u}\right), \dot{z}_{s, u}\right\rangle\right) \mathrm{d} s\right\} \\
\geq & \inf _{u \in V}\left\{\int _ { 0 } ^ { t } \mathrm { e } ^ { - \lambda s } \left(-H\left(x_{s, u}, z_{s, u}, \nabla_{x} v\left(x_{s, u}, z_{s, u}\right)\right)-\lambda v\left(x_{s, u}, z_{s, u}\right)\right.\right. \\
& \left.\left.-\left\langle D_{z} v\left(x_{s, u}, z_{s, u}\right), \dot{z}_{s, u}\right\rangle\right) \mathrm{d} s\right\} .
\end{aligned}
$$

It is natural to expect the integrand above to converge as $t \rightarrow 0^{+}$, uniformly in $u$ to

$$
-H\left(x, z, \nabla_{x} v(x, z)\right)-\lambda v(x, z)-\left\langle D_{z} v(x, z), \dot{z}\right\rangle
$$

so that:

$$
\lambda v(x, z)+\left\langle D_{z} v(x, z), \dot{z}\right\rangle+H\left(x, z, \nabla_{x} v(x, z)\right) \geq 0 .
$$

Thus, at least formally, the Hamilton-Jacobi-Bellman equation satisfied by the value function $v$ can be written as:

$$
\lambda v(x, z)+H\left(x, z, \nabla_{x} v(x, z)\right)+\left\langle D_{z} v(x, z), \dot{z}\right\rangle=0 \text { on } E_{0}
$$

with $H$ defined by (4.5).

The next lemma whose easy proof is left to the reader gives the regularity properties of $H$ :

Lemma 4.3. Let $H$ be the Hamiltonian defined by (4.5). Assume that (H1), (H2) and (H3) hold, then there exists a nonnegative constant $C$ such that:

$$
|H(x, z, p)-H(y, w, p)| \leq C\left(|x-y|+\|z-w\|_{L^{2}}\right)(1+|p|),
$$

and

$$
|H(x, z, p)-H(x, z, q)| \leq C|p-q|\left(1+|x|+\|z\|_{L^{2}}\right),
$$

for every $(x, z, y, w, p, q) \in\left(\mathbb{R}^{d} \times L^{2}\right)^{2} \times \mathbb{R}^{d} \times \mathbb{R}^{d}$. If, in addition (H'2) is satisfied, then (4.7) can be improved by:

$$
|H(x, z, p)-H(y, w, p)| \leq C\left(|x-y|+\|z-w\|_{\left(H^{1}\right)^{\prime}}\right)(1+|p|),
$$

for every $(x, z, y, w, p) \in\left(\mathbb{R}^{d} \times L^{2}\right)^{2} \times \mathbb{R}^{d}$.

\subsection{Definition of viscosity solutions}

The formal manipulations above actually suggest that the natural definition of viscosity solutions in the present context should read as:

Definition 4.1. Let $w \in \mathrm{BUC}\left(\mathbb{R}^{\mathrm{d}} \times \mathrm{L}^{2}, \mathbb{R}\right) \cap \mathrm{C}^{0}\left(\mathbb{R}^{\mathrm{d}} \times \mathrm{L}_{\mathrm{w}}^{2}, \mathbb{R}\right)$, then $w$ is said to be:

(1) A viscosity subsolution of (4.6) on $\mathbb{R}^{d} \times L^{2}$ if for every $\left(x_{0}, z_{0}\right) \in \mathbb{R}^{d} \times L^{2}$ and every $\phi \in C^{1}\left(\mathbb{R}^{d} \times L^{2}, \mathbb{R}\right)$ such that $w-\phi$ has a local maximum (in the sense of the strong topology of $\mathbb{R}^{d} \times L^{2}$ ) at $\left(x_{0}, z_{0}\right)$, one has:

$$
\lambda w\left(x_{0}, z_{0}\right)+H\left(x_{0}, z_{0}, \nabla_{x} \phi\left(x_{0}, z_{0}\right)\right)+\liminf _{(x, z) \in E_{0} \rightarrow\left(x_{0}, z_{0}\right)}\left\langle D_{z} \phi(x, z), \dot{z}\right\rangle \leq 0
$$

where the convergence $(x, z) \in E_{0} \rightarrow\left(x_{0}, z_{0}\right)$ has to be understood in the strong $\mathbb{R}^{d} \times L^{2}$ sense; 
(2) A viscosity supersolution of (4.6) on $\mathbb{R}^{d} \times L^{2}$ if for every $\left(x_{0}, z_{0}\right) \in \mathbb{R}^{d} \times L^{2}$ and every $\phi \in C^{1}\left(\mathbb{R}^{d} \times L^{2}, \mathbb{R}\right)$ such that $w-\phi$ has a local minimum (in the sense of the strong topology of $\mathbb{R}^{d} \times L^{2}$ ) at $\left(x_{0}, z_{0}\right)$, one has:

$$
\lambda w\left(x_{0}, z_{0}\right)+H\left(x_{0}, z_{0}, \nabla_{x} \phi\left(x_{0}, z_{0}\right)\right)+\limsup _{(x, z) \in E_{0} \rightarrow\left(x_{0}, z_{0}\right)}\left\langle D_{z} \phi(x, z), \dot{z}\right\rangle \geq 0
$$

where the convergence $(x, z) \in E_{0} \rightarrow\left(x_{0}, z_{0}\right)$ has to be understood in the strong $\mathbb{R}^{d} \times L^{2}$ sense;

(3) A viscosity solution of (4.6) on $\mathbb{R}^{d} \times L^{2}$ if it is both a viscosity subsolution of (4.6) and a viscosity supersolution of (4.6) on $\mathbb{R}^{d} \times L^{2}$.

Let us mention that the previous definition is not exactly the classical one of Crandall and Lions: here we allow a larger class of test-functions and use liminf and limsup to handle the unbounded term in the equation whereas Crandall and Lions use a smaller class of test-functions for which the evaluation of the unbounded term evaluated at the gradient directly makes sense. The reason for the present choice is that it will simplify the proof of the comparison principle without making the proof of the fact that the value function is a viscosity solution difficult. In principle, if one considers too large classes of test-functions, existence becomes harder to prove (and may even be a real problem), but as we will see in the next paragraph, existence here will be guaranteed by the fact that the value function is a viscosity solution.

\subsection{The value function is a viscosity solution}

Proposition 4.1. Assume that (H1), (H2) and (H3) hold. The value function $v$ defined by (2.5) is a viscosity solution of (4.6) on $\mathbb{R}^{d} \times L^{2}$.

Proof. Step 1. $v$ is a viscosity subsolution.

Let $\alpha_{0}:=\left(x_{0}, z_{0}\right) \in \mathbb{R}^{d} \times L^{2}$ and $\phi \in C^{1}\left(\mathbb{R}^{d} \times L^{2}, \mathbb{R}\right)$ such that $v\left(x_{0}, z_{0}\right)=\phi\left(x_{0}, z_{0}\right)$ and $\phi \geq v$ on the ball $B_{r}:=B\left(\left(x_{0}, z_{0}\right), r\right)$ of $\mathbb{R}^{d} \times L^{2}$. Let $u \in K$ be some constant control. There exists $\varepsilon_{0}>0$ such that for all $\varepsilon \in\left(0, \varepsilon_{0}\right)$, there is some $\alpha_{\varepsilon}:=\left(x_{\varepsilon}, z_{\varepsilon}\right) \in E_{0}$ such that

$$
\phi\left(\alpha_{\varepsilon}\right)-\varepsilon^{2} \leq v\left(\alpha_{\varepsilon}\right) \leq \phi\left(\alpha_{\varepsilon}\right), \lim _{\varepsilon} \alpha_{\varepsilon}=\alpha_{0} \text { in } \mathbb{R}^{d} \times L^{2}
$$

and such that $\alpha_{\varepsilon, s}=\left(x_{\varepsilon, s}, z_{\varepsilon, s}\right)=\left(y_{\alpha_{\varepsilon}, u}(s), y_{\alpha_{\varepsilon}, u}(s-).\right)$ belongs to $B_{r}$ for all $s \in[0, \varepsilon]$. Note that by construction, $\alpha_{\varepsilon, s}$ belongs to $E_{0}$ for every $s \in[0, \varepsilon]$. The dynamic programming principle first yields

$$
\phi\left(\alpha_{\varepsilon}\right)-\varepsilon^{2} \leq \int_{0}^{\varepsilon} \mathrm{e}^{-\lambda s} L\left(x_{\varepsilon, s}, u\right) \mathrm{d} s+\mathrm{e}^{-\lambda \varepsilon} \phi\left(\alpha_{\varepsilon, \varepsilon}\right) .
$$

Thanks to the smoothness of $\phi$, Lemma 4.2 and Remark 4.1, we can write:

$$
\mathrm{e}^{-\lambda \varepsilon} \phi\left(\alpha_{\varepsilon, \varepsilon}\right)=\phi\left(\alpha_{\varepsilon}\right)+\int_{0}^{\varepsilon} \mathrm{e}^{-\lambda s}\left(\nabla_{x} \phi\left(\alpha_{\varepsilon, s}\right) \cdot \mathcal{F}\left(\alpha_{\varepsilon, s}, u\right)-\lambda \phi\left(\alpha_{\varepsilon, s}\right)\right) \mathrm{d} s-\int_{0}^{\varepsilon} \mathrm{e}^{-\lambda s}\left\langle D_{z} \phi\left(\alpha_{\varepsilon, s}\right), \dot{z}_{\varepsilon, s}\right\rangle \mathrm{d} s .
$$

Since $\alpha_{\varepsilon}$ converges to $\alpha_{0}$, one easily deduces from Lemma 4.1 that

$$
\sup _{s \in[0, \varepsilon]}\left\|\alpha_{\varepsilon, s}-\alpha_{0}\right\|_{\mathbb{R}^{d} \times L^{2}} \rightarrow 0 \text { as } \varepsilon \rightarrow 0^{+} .
$$

Denoting by $\psi(s, \alpha):=\mathrm{e}^{-\lambda s}\left(\left(\nabla_{x} \phi(\alpha) \cdot \mathcal{F}(\alpha, u)-\lambda \phi(\alpha)\right)\right.$, we then have

$$
\sup _{s \in[0, \varepsilon]}\left|\psi\left(s, \alpha_{\varepsilon, s}\right)-\psi\left(0, \alpha_{\varepsilon}\right)\right| \rightarrow 0 \text { as } \varepsilon \rightarrow 0^{+} .
$$


We thus get:

$$
\mathrm{e}^{-\lambda \varepsilon} \phi\left(\alpha_{\varepsilon, \varepsilon}\right)=\phi\left(\alpha_{\varepsilon}\right)+\varepsilon\left(\nabla_{x} \phi\left(\alpha_{\varepsilon}\right) \cdot \mathcal{F}\left(\alpha_{\varepsilon}, u\right)-\lambda \phi\left(\alpha_{\varepsilon}\right)-\int_{0}^{\varepsilon} \mathrm{e}^{-\lambda s}\left\langle D_{z} \phi\left(\alpha_{\varepsilon, s}\right), \dot{z}_{\varepsilon, s}\right\rangle \mathrm{d} s\right)+o(\varepsilon) .
$$

Using (4.10), dividing by $\varepsilon$ and taking the liminf as $\varepsilon \rightarrow 0^{+}$, we then get:

$$
\begin{aligned}
& 0 \geq \liminf _{\varepsilon \rightarrow 0^{+}}\left(-\frac{1}{\varepsilon} \int_{0}^{\varepsilon} \mathrm{e}^{-\lambda s} L\left(x_{\varepsilon, s}, u\right) \mathrm{d} s-\nabla_{x} \phi\left(\alpha_{\varepsilon}\right) \cdot \mathcal{F}\left(\alpha_{\varepsilon}, u\right)+\lambda \phi\left(\alpha_{\varepsilon}\right)\right) \\
& \quad+\liminf _{\varepsilon \rightarrow 0^{+}} \frac{1}{\varepsilon} \int_{0}^{\varepsilon} \mathrm{e}^{-\lambda s}\left\langle D_{z} \phi\left(\alpha_{\varepsilon, s}\right), \dot{z}_{\varepsilon, s}\right\rangle \mathrm{d} s .
\end{aligned}
$$

Since we have:

$$
\liminf _{\varepsilon \rightarrow 0^{+}} \frac{1}{\varepsilon} \int_{0}^{\varepsilon} \mathrm{e}^{-\lambda s}\left\langle D_{z} \phi\left(\alpha_{\varepsilon, s}\right), \dot{z}_{\varepsilon, s}\right\rangle \mathrm{d} s \geq \liminf _{(x, z) \in E_{0} \rightarrow \alpha_{0}}\left\langle D_{z} \phi(x, z), \dot{z}\right\rangle,
$$

we then obtain:

$$
0 \geq-L\left(x_{0}, u\right)-\nabla_{x} \phi\left(\alpha_{0}\right) \cdot \mathcal{F}\left(\alpha_{0}, u\right)+\lambda v\left(\alpha_{0}\right)+\liminf _{(x, z) \in E_{0} \rightarrow \alpha_{0}}\left\langle D_{z} \phi(x, z), \dot{z}\right\rangle .
$$

Since $u \in K$ is arbitrary in the previous inequality, taking the supremum with respect to $u$ and using the very definition of $H$ given in (4.5), we thus deduce:

$$
\lambda v\left(\alpha_{0}\right)+H\left(\alpha_{0}, \nabla_{x} \phi\left(\alpha_{0}\right)\right)+\liminf _{(x, z) \in E_{0} \rightarrow \alpha_{0}}\left\langle D_{z} \phi(x, z), \dot{z}\right\rangle \leq 0
$$

which proves that $v$ is a viscosity subsolution of (4.6) on $\mathbb{R}^{d} \times L^{2}$.

Step 2. $v$ is a viscosity supersolution.

Now let $\phi \in C^{1}\left(\mathbb{R}^{d} \times L^{2}, \mathbb{R}\right)$ be such that $v\left(\alpha_{0}\right)=\phi\left(\alpha_{0}\right)$ and $v \geq \phi$ on the ball $B_{r}:=B\left(\alpha_{0}, r\right)$ of $\mathbb{R}^{d} \times L^{2}$. There exists $\varepsilon_{0}>0$ such that for all $\varepsilon \in\left(0, \varepsilon_{0}\right)$, there is some $\alpha_{\varepsilon}:=\left(x_{\varepsilon}, z_{\varepsilon}\right) \in E_{0}$ such that

$$
\phi\left(\alpha_{\varepsilon}\right)+\varepsilon^{2} \geq v\left(\alpha_{\varepsilon}\right) \geq \phi\left(\alpha_{\varepsilon}\right), \lim _{\varepsilon} \alpha_{\varepsilon}=\alpha_{0} \text { in } \mathbb{R}^{d} \times L^{2}
$$

and such that for every $u \in V, \alpha_{\varepsilon, s, u}=\left(x_{\varepsilon, s, u}, z_{\varepsilon, s, u}\right)=\left(y_{\alpha_{\varepsilon}, u}(s), y_{\alpha_{\varepsilon}, u}(s-).\right)$ belongs to $B_{r}$ for all $s \in[0, \varepsilon]$. The dynamic programming principle then gives:

$$
\phi\left(\alpha_{\varepsilon}\right)+\varepsilon^{2} \geq \inf _{u \in V}\left\{\int_{0}^{\varepsilon} \mathrm{e}^{-\lambda s} L\left(x_{\varepsilon, s, u}, u(s)\right) \mathrm{d} s+\mathrm{e}^{-\lambda \varepsilon} \phi\left(\alpha_{\varepsilon, \varepsilon, u}\right)\right\}
$$

With Lemma 4.2 and Remark 4.1, we can rewrite

$$
\mathrm{e}^{-\lambda \varepsilon} \phi\left(\alpha_{\varepsilon, \varepsilon, u}\right)=\phi\left(\alpha_{\varepsilon}\right)-\int_{0}^{\varepsilon} \mathrm{e}^{-\lambda s} \lambda \phi\left(\alpha_{\varepsilon, s, u}\right) \mathrm{d} s+\int_{0}^{\varepsilon} \mathrm{e}^{-\lambda s}\left(\nabla_{x} \phi\left(\alpha_{\varepsilon, s, u}\right) \cdot \mathcal{F}\left(\alpha_{\varepsilon, s, u}, u(s)\right)-\left\langle D_{z} \phi\left(\alpha_{\varepsilon, s, u}\right), \dot{z}_{\varepsilon, s, u}\right\rangle\right) \mathrm{d} s .
$$

Using (4.11), we then get:

$$
\varepsilon^{2} \geq \inf _{u \in V}\left\{\int_{0}^{\varepsilon} \mathrm{e}^{-\lambda s}\left(L\left(x_{\varepsilon, s, u}, u(s)\right)-\lambda \phi\left(\alpha_{\varepsilon, s, u}\right)+\nabla_{x} \phi\left(\alpha_{\varepsilon, s, u}\right) \cdot \mathcal{F}\left(\alpha_{\varepsilon, s, u}, u(s)\right)-\left\langle D_{z} \phi\left(\alpha_{\varepsilon, s, u}\right), \dot{z}_{\varepsilon, s, u}\right\rangle\right) \mathrm{d} s\right\}
$$


and then

$$
\varepsilon^{2} \geq \inf _{u \in V}\left\{\int_{0}^{\varepsilon} \mathrm{e}^{-\lambda s}\left(-H\left(\alpha_{\varepsilon, s, u}, \nabla_{x} \phi\left(\alpha_{\varepsilon, s, u}\right)\right)-\lambda \phi\left(\alpha_{\varepsilon, s, u}\right)-\left\langle D_{z} \phi\left(\alpha_{\varepsilon, s, u}\right), \dot{z}_{\varepsilon, s, u}\right\rangle\right) \mathrm{d} s\right\}
$$

From the continuity of $\nabla_{x} \phi$ and $H$, we deduce the (uniform in $u$ ) convergence as $\varepsilon \rightarrow 0^{+}$and $s \in[0, \varepsilon]$, of $\nabla_{x} \phi\left(\alpha_{\varepsilon, s, u}\right)$, and $H\left(\alpha_{\varepsilon, s, u}, \nabla_{x} \phi\left(\alpha_{\varepsilon, s, u}\right)\right)$ respectively to $\nabla_{x} \phi\left(\alpha_{0}\right)$ and $H\left(\alpha_{0}, \nabla_{x} \phi\left(\alpha_{0}\right)\right)$. Using the fact that

$$
\limsup _{\varepsilon \rightarrow 0^{+}} \sup _{u \in V}\left\{\frac{1}{\varepsilon} \int_{0}^{\varepsilon}\left\langle D_{z} \phi\left(\alpha_{\varepsilon, s, u}\right), \dot{z}_{\varepsilon, s, u}\right\rangle \mathrm{d} s\right\} \leq \limsup _{(x, z) \in E_{0} \rightarrow \alpha_{0}}\left\langle D_{z} \phi(x, z), \dot{z}\right\rangle
$$

dividing by $-\varepsilon$ inequality (4.12) and taking the limsup as $\varepsilon \rightarrow 0^{+}$, we thus get:

$$
\lambda v\left(\alpha_{0}\right)+H\left(\alpha_{0}, \nabla_{x} \phi\left(\alpha_{0}\right)\right)+\limsup _{(x, z) \in E_{0} \rightarrow \alpha_{0}}\left\langle D_{z} \phi(x, z), \dot{z}\right\rangle \geq 0
$$

which proves that $v$ is a viscosity supersolution of (4.6) on $\mathbb{R}^{d} \times L^{2}$.

\section{Comparison And uniqueness}

\subsection{Preliminaries}

Our aim now is to prove that $v$ is the unique viscosity subsolution of the Hamilton-Jacobi-Bellman equation:

$$
\lambda v(x, z)+H\left(x, z, \nabla_{x} v(x, z)\right)+\left\langle D_{z} v(x, z), \dot{z}\right\rangle=0
$$

This will of course follow from a comparison result stating that if $v_{1}$ and $v_{2}$ (in a suitable class of continuous functions) are respectively a viscosity subsolution and a viscosity supersolution of the equation then $v_{1} \leq v_{2}$ on $E:=\mathbb{R}^{d} \times L^{2}$. As usual, the comparison result is proved, by introducing a doubling of variables and by considering perturbed problems of the form:

$$
\sup \left\{v_{1}\left(\alpha_{1}\right)-v_{2}\left(\alpha_{2}\right)-P_{\theta}\left(\alpha_{1}, \alpha_{2}\right):\left(\alpha_{1}, \alpha_{2}\right) \in\left(\mathbb{R}^{d} \times L^{2}\right)^{2}\right\}
$$

where $P_{\theta}$ is some perturbation function (depending on small parameters $\theta$ ). This perturbation includes a penalization of the doubling of variables and coercive terms that ensure the existence of maxima say $\alpha_{1}^{\theta}$ and $\alpha_{2}^{\theta}$. Then one uses the fact that $v_{1}$ is a viscosity subsolution by taking $\phi:=P_{\theta}\left(., \alpha_{2}^{\theta}\right)$ as test-function.

To overcome the difficulties due to the fact that the term $\left\langle D_{z} \phi(\alpha), \dot{z}\right\rangle$ is only defined for $z \in H^{1}$ and that the equation is only justified when in addition $z(0)=x$, one has to be careful on the choice of the perturbation $P_{\theta}$. For general infinite-dimensional Hamilton-Jacobi equations with an unbounded linear term, these difficulties were solved in a general way by Crandall and Lions in [11-13]. One of the key arguments of Crandall and Lions in [11] is to use a suitable norm to penalize the doubling of variables. In our context this, roughly speaking, amounts to use a kind of $\left(H^{1}\right)^{\prime}$ norm instead of the $L^{2}$ norm in the doubling of variables. As usual, the comparison proof will very much rely on the use of quadratic test-functions of the form:

$$
\phi(\alpha)=\Phi_{1}(x)+\Phi_{2}(\alpha)+\Phi_{3}(z):=a|x|^{2}+b\left\langle B\left(\alpha-\alpha_{0}\right), \alpha-\alpha_{0}\right\rangle+c\|z\|^{2}
$$

where $a$ and $b$ and $c$ are constants and $B$ is a bounded positive self-adjoint operator of $\mathbb{R}^{d} \times L^{2}$. Let us first note that in our case the term $\left\langle\Phi_{3}^{\prime}(z), \dot{z}\right\rangle=2 c\langle z, \dot{z}\rangle$ can be dealt easily since, if $z \in H^{1}$, one has:

$$
\langle z, \dot{z}\rangle=-\frac{1}{2}|z(0)|^{2}
$$


and since in the definition of viscosity solutions, we have imposed the convergence of the initial value $z(0)$, it is easy to figure out that this term won't be a big problem in the proof. The difficulty of dealing with the second term $\left\langle D_{z} \Phi_{2}(\alpha), \dot{z}\right\rangle$ can be solved by properly choosing $B$ as in Crandall and Lions [12] who emphasized the good properties $B$ should enjoy for the comparison proof to work. We now proceed to the explicit construction of such a $B$ in our context.

Let us endow $E:=\mathbb{R}^{d} \times L^{2}$ with its standard Hilbertian structure, i.e. with the norm:

$$
\|\alpha\|^{2}:=|x|^{2}+\|z\|_{L^{2}}^{2}, \forall \alpha=(x, z) \in E
$$

and the corresponding inner product $\langle.,$.$\rangle . Let T$ be the linear unbounded operator on $E$ with domain $D(T)=\mathbb{R}^{d} \times H^{1}$ and defined by

$$
T(y, w):=(y-w(0),-\dot{w}), \forall(y, w) \in D(T) .
$$

Its adjoint $T^{*}$ has domain $D\left(T^{*}\right)=E_{0}=\left\{(x, z) \in E: z \in H^{1}, z(0)=x\right\}$ and is given by

$$
T^{*}(x, z):=(z(0), \dot{z})=(x, \dot{z}), \forall(x, z) \in D\left(T^{*}\right)=E_{0} .
$$

The unbounded operator $I+T^{*} T$ therefore has domain

$$
D\left(T^{*} T\right)=\left\{(y, w) \in E: w \in H^{2}, y=w(0)-\dot{w}(0)\right\}
$$

and is given by

$$
\left(I+T^{*} T\right)(y, w):=(2 y-w(0),-\ddot{w}+w), \forall(y, w) \in D\left(T^{*} T\right) .
$$

Now, let us set $B:=\left(I+T^{*} T\right)^{-1}$. For $\alpha=(x, z) \in E,(y, w):=B(\alpha)$ is defined as follows: firstly, $w \in H^{2}$ is the solution of

secondly, $y$ is defined by

$$
\left\{\begin{array}{ccc}
-\ddot{w}+w & = & z \\
-2 \dot{w}(0)+w(0) & = & x
\end{array} \text { in }(0,+\infty)\right.
$$

$$
y=\frac{x+w(0)}{2}=w(0)-\dot{w}(0) .
$$

In the sequel we shall also denote $B=\left(B_{1}, B_{2}\right)$ where $B_{2}(x, z)=w$ is defined by $(5.4)$ and $B_{1}(x, z)=y$ is given by (5.4) and (5.5). Setting:

$$
\|\alpha\|_{B}^{2}:=\langle B(\alpha), \alpha\rangle, \forall \alpha=(x, z) \in E,
$$

and defining $w:=B_{2}(\alpha)$ by (5.4), an elementary computation shows that

$$
\|\alpha\|_{B}^{2}=\frac{|x|^{2}}{2}+\frac{|w(0)|^{2}}{2}+\|w\|_{H^{1}}^{2} .
$$

Now, using the fact that $\|z\|_{\left(H^{1}\right)^{\prime}}^{2}=\|w+\varphi\|_{H^{1}}^{2}$ where

$$
\left\{\begin{array}{ccc}
-\ddot{\varphi}+\varphi & = & 0 \\
\dot{\varphi}(0) & = & \frac{x-w(0)}{2},
\end{array} \quad \text { in }(0,+\infty)\right.
$$

we then have for some constant $m \geq 0$ :

$$
\|z\|_{\left(H^{1}\right)^{\prime}}^{2} \leq 2\left(\|w\|_{H^{1}}^{2}+\|\varphi\|_{H^{1}}^{2}\right) \leq 2\|w\|_{H^{1}}^{2}+m\left(|x|^{2}+|w(0)|^{2}\right)
$$

and thus one deduces from (5.6) that there is some constant $C>0$, such that

$$
\|(x, z)\|_{B}^{2} \geq C\left(|x|^{2}+\|z\|_{\left(H^{1}\right)^{\prime}}^{2}\right), \forall(x, z) \in E .
$$


Obviously, by construction $B$ is a self-adjoint, nonnegative compact operator on $E$ and $T B$ is a bounded operator on $E$. For $\alpha=(x, z) \in E_{0}=D\left(T^{*}\right)$ and $w:=B_{2}(\alpha)$, some computations lead to:

$$
\langle T B(\alpha), \alpha\rangle=\left\langle B(\alpha), T^{*}(\alpha)\right\rangle=\frac{3}{8}|x-w(0)|^{2}+\frac{x \cdot w(0)}{2} \geq \frac{1}{8}|x-w(0)|^{2},
$$

and since $T B$ is continuous and $E_{0}$ is dense in $E$ this proves

$$
\langle T B(\alpha), \alpha\rangle \geq 0, \forall \alpha \in E .
$$

Let us also remark that for $\alpha \in E$ and $\beta:=B(\alpha)$ one has:

$$
\|\alpha\|_{B}^{2}=\langle\alpha, \beta\rangle=\left\langle\left(I+T^{*} T\right)(\beta), \beta\right\rangle \geq\|\beta\|^{2},
$$

so that

$$
\|B(\alpha)\| \leq\|\alpha\|_{B}, \forall \alpha \in E
$$

In the sequel we will denote by $\mathrm{BUC}\left(\mathrm{E}_{\mathrm{B}}, \mathbb{R}\right)$ the space of bounded and uniformly continuous functions on $E$ equipped with the norm $\|.\|_{B}$. Let us remark that because of (5.7), bounded functions which are uniformly continuous on $E$ equipped with the usual norm of $\mathbb{R}^{d} \times\left(H^{1}\right)^{\prime}$ belong to $\mathrm{BUC}\left(\mathrm{E}_{\mathrm{B}}, \mathbb{R}\right)$. In particular if $A$ is $H^{1}$ then the value function $v$ defined by $(2.5)$ belongs to $\mathrm{BUC}\left(\mathrm{E}_{\mathrm{B}}, \mathbb{R}\right)$.

We end this paragraph by remarking that equation (4.6) now can (at least formally) be rewritten as

$$
\lambda v(\alpha)+H\left(\alpha, \nabla_{x} v(\alpha)\right)-x \cdot \nabla_{x} v(\alpha)+\left\langle T^{*}(\alpha), D v(\alpha)\right\rangle=0, \alpha \in D\left(T^{*}\right) .
$$

As in Crandall and Lions [12], we will take advantage of this structure (where $\left\langle T^{*}(\alpha), D v(\alpha)\right\rangle$ has to be understood as $\langle\alpha, T(D v(\alpha))\rangle)$ by imposing restrictions on test-functions (typically of the form $\left\|\alpha-\alpha_{0}\right\|_{B}^{2}$ ) rather than on $\alpha$.

\subsection{Comparison theorem}

The comparison result for (4.6) then reads as:

Theorem 5.1. Assume that (H1), (H'2) and (H3) hold. Let $v_{1}$ and $v_{2}$ be in $\mathrm{BUC}\left(\mathrm{E}_{\mathrm{B}}, \mathbb{R}\right) \cap \mathrm{C}^{0}\left(\mathrm{E}_{\mathrm{w}}, \mathbb{R}\right)$ respectively a viscosity subsolution and a viscosity supersolution of (4.6) on $\mathbb{R}^{d} \times L^{2}$ then $v_{1} \leq v_{2}$ on $\mathbb{R}^{d} \times L^{2}$.

Proof. Let us define $M:=\sup _{E}\left(v_{1}-v_{2}\right), B:=\left(I+T^{*} T\right)^{-1}$ and

$$
\|\alpha\|_{B}^{2}:=\langle B(\alpha), \alpha\rangle, \forall \alpha=(x, z) \in E
$$

as before. For all $\varepsilon>0, \delta>0$ and $\alpha_{1}:=\left(x_{1}, z_{1}\right), \alpha_{2}:=\left(x_{2}, z_{2}\right)$, in $\mathbb{R}^{d} \times L^{2}$, let us set $\theta:=(\varepsilon, \delta)$ and

$$
\Phi_{\theta}\left(\alpha_{1}, \alpha_{2}\right):=v_{1}\left(\alpha_{1}\right)-v_{2}\left(\alpha_{2}\right)-\frac{1}{2 \varepsilon}\left\|\alpha_{1}-\alpha_{2}\right\|_{B}^{2}-\frac{\delta}{2}\left(\left\|\alpha_{1}\right\|^{2}+\left\|\alpha_{2}\right\|^{2}\right) .
$$

Let us also define:

$$
M_{\varepsilon, \delta}=M_{\theta}=\sup \left\{\Phi_{\theta}\left(\alpha_{1}, \alpha_{2}\right),\left(\alpha_{1}, \alpha_{2}\right) \in E \times E\right\} .
$$

The weak continuity and boundedness properties of $v_{1}, v_{2}$ ensure that the supremum $M_{\theta}$ is attained at some points $\alpha_{i}^{\theta}=\left(x_{i}^{\theta}, z_{i}^{\theta}\right)$ for $i=1,2$.

Let us set

we then have:

$$
\Phi_{1}(\alpha):=\frac{1}{2 \varepsilon}\left\|\alpha-\alpha_{2}^{\theta}\right\|_{B}^{2}+\frac{\delta}{2}\|\alpha\|^{2}, \forall \alpha \in E,
$$

$$
D \Phi_{1}(\alpha)=\left(\frac{1}{\varepsilon} B_{1}\left(\alpha-\alpha_{2}^{\theta}\right)+\delta x, \frac{1}{\varepsilon} B_{2}\left(\alpha-\alpha_{2}^{\theta}\right)+\delta z\right), \forall \alpha=(x, z) \in E .
$$


Since $\alpha_{1}^{\theta}$ is a maximum of $v_{1}-\Phi_{1}$ on $E$ and since $v_{1}$ is a viscosity subsolution of (4.6), we get from Definition 4.1:

$$
\lambda v_{1}\left(\alpha_{1}^{\theta}\right)+H\left(\alpha_{1}^{\theta}, q_{\theta}+\delta x_{1}^{\theta}\right)+\liminf _{\alpha=(x, z) \in E_{0} \rightarrow \alpha_{1}^{\theta}}\left\langle\frac{1}{\varepsilon} B_{2}\left(\alpha-\alpha_{2}^{\theta}\right)+\delta z, \dot{z}\right\rangle \leq 0,
$$

where

We then remark that

$$
q_{\theta}:=\frac{1}{\varepsilon} B_{1}\left(\alpha_{1}^{\theta}-\alpha_{2}^{\theta}\right)
$$

Next, we write:

$$
\langle z, \dot{z}\rangle=-\frac{1}{2}|z(0)|^{2} \rightarrow-\frac{1}{2}\left|x_{1}^{\theta}\right|^{2} \text { as } \alpha \in E_{0} \rightarrow \alpha_{1}^{\theta} .
$$

$$
\begin{aligned}
\left\langle B_{2}\left(\alpha-\alpha_{2}^{\theta}\right), \dot{z}\right\rangle & =\left\langle B\left(\alpha-\alpha_{2}^{\theta}\right), T^{*}(\alpha)\right\rangle-B_{1}\left(\alpha-\alpha_{2}^{\theta}\right) \cdot x \\
& =\left\langle T B\left(\alpha-\alpha_{2}^{\theta}\right), \alpha\right\rangle-B_{1}\left(\alpha-\alpha_{2}^{\theta}\right) \cdot x
\end{aligned}
$$

and since $T B$ and $B_{1}$ are continuous, we get:

$$
\left\langle B_{2}\left(\alpha-\alpha_{2}^{\theta}\right), \dot{z}\right\rangle \rightarrow\left\langle T B\left(\alpha_{1}^{\theta}-\alpha_{2}^{\theta}\right), \alpha_{1}^{\theta}\right\rangle-B_{1}\left(\alpha_{1}^{\theta}-\alpha_{2}^{\theta}\right) \cdot x_{1}^{\theta} \text { as } \alpha \in E_{0} \rightarrow \alpha_{1}^{\theta} .
$$

Hence (5.11) can be rewritten as:

$$
\lambda v_{1}\left(\alpha_{1}^{\theta}\right)+H\left(\alpha_{1}^{\theta}, q_{\theta}+\delta x_{1}^{\theta}\right)-q_{\theta} \cdot x_{1}^{\theta}-\frac{\delta}{2}\left|x_{1}^{\theta}\right|^{2}+\frac{1}{\varepsilon}\left\langle T B\left(\alpha_{1}^{\theta}-\alpha_{2}^{\theta}\right), \alpha_{1}^{\theta}\right\rangle \leq 0 .
$$

Using in a similar way the fact that $v_{2}$ is a viscosity supersolution, we arrive at:

$$
\lambda v_{2}\left(\alpha_{2}^{\theta}\right)+H\left(\alpha_{2}^{\theta}, q_{\theta}-\delta x_{2}^{\theta}\right)-q_{\theta} \cdot x_{2}^{\theta}+\frac{\delta}{2}\left|x_{2}^{\theta}\right|^{2}-\frac{1}{\varepsilon}\left\langle T B\left(\alpha_{2}^{\theta}-\alpha_{1}^{\theta}\right), \alpha_{2}^{\theta}\right\rangle \geq 0 .
$$

Subtracting (5.13) and (5.14) then yields:

$$
\begin{aligned}
\lambda\left(v_{1}\left(\alpha_{1}^{\theta}\right)-v_{2}\left(\alpha_{2}^{\theta}\right)\right)+H\left(\alpha_{1}^{\theta}, q_{\theta}+\delta x_{1}^{\theta}\right)-H & \left(\alpha_{2}^{\theta}, q_{\theta}-\delta x_{2}^{\theta}\right) \\
& +q_{\theta} \cdot\left(x_{2}^{\theta}-x_{1}^{\theta}\right)-\frac{\delta}{2}\left(\left|x_{1}^{\theta}\right|^{2}+\left|x_{1}^{\theta}\right|^{2}\right)+\frac{1}{\varepsilon}\left\langle T B\left(\alpha_{1}^{\theta}-\alpha_{2}^{\theta}\right), \alpha_{1}^{\theta}-\alpha_{2}^{\theta}\right\rangle \leq 0 .
\end{aligned}
$$

Now, thanks to (5.8), the last term is nonnegative, which gives:

$$
\lambda\left(v_{1}\left(\alpha_{1}^{\theta}\right)-v_{2}\left(\alpha_{2}^{\theta}\right)\right)+H\left(\alpha_{1}^{\theta}, q_{\theta}+\delta x_{1}^{\theta}\right)-H\left(\alpha_{2}^{\theta}, q_{\theta}-\delta x_{2}^{\theta}\right)+q_{\theta} \cdot\left(x_{2}^{\theta}-x_{1}^{\theta}\right) \leq \frac{\delta}{2}\left(\left|x_{1}^{\theta}\right|^{2}+\left|x_{1}^{\theta}\right|^{2}\right) .
$$

Thanks to the fact that $v_{1}$ and $v_{2}$ belong to $\mathrm{BUC}\left(\mathrm{E}_{\mathrm{B}}, \mathbb{R}\right)$, by standard arguments, following exactly the proof of Lemma 2.9 in [4] (see also [3]), one easily obtains:

$$
\lim _{\theta \rightarrow\left(0^{+}, 0^{+}\right)}\left(\delta\left\|\alpha_{i}^{\theta}\right\|^{2}, \varepsilon^{-1}\left\|\alpha_{1}^{\theta}-\alpha_{2}^{\theta}\right\|_{B}^{2}, M_{\theta}, v_{1}\left(\alpha_{1}^{\theta}\right)-v_{2}\left(\alpha_{2}^{\theta}\right)\right)=(0,0, M, M) .
$$

On the one hand, using (5.6) and (5.16), we have:

$$
\left|x_{1}^{\theta}-x_{2}^{\theta}\right| \leq \sqrt{2}\left\|\alpha_{1}^{\theta}-\alpha_{2}^{\theta}\right\|_{B}=o(\sqrt{\varepsilon})
$$

on the other hand, using (5.9), we have

$$
\left|q_{\theta}\right| \leq \frac{1}{\varepsilon}\left\|B\left(\alpha_{1}^{\theta}-\alpha_{2}^{\theta}\right)\right\| \leq \frac{1}{\varepsilon}\left\|\alpha_{1}^{\theta}-\alpha_{2}^{\theta}\right\|_{B}=o\left(\frac{1}{\sqrt{\varepsilon}}\right)
$$


so that

$$
\lim _{\theta \rightarrow\left(0^{+}, 0^{+}\right)} q_{\theta} \cdot\left(x_{2}^{\theta}-x_{1}^{\theta}\right)=0 .
$$

Lemma 4.3 and (5.7) then imply that there is a nonnegative constant $C$ such that:

$$
|H(\alpha, p)-H(\beta, p)| \leq C\|\alpha-\beta\|_{B}(1+|p|), \forall(\alpha, \beta, p) \in E^{2} \times \mathbb{R}^{d},
$$

and

We thus deduce

$$
|H(\alpha, p)-H(\alpha, q)| \leq C|p-q|(1+\|\alpha\|), \forall(\alpha, p, q) \in E \times \mathbb{R}^{d} \times \mathbb{R}^{d}
$$

$$
\begin{aligned}
H\left(\alpha_{1}^{\theta}, q_{\theta}+\delta x_{1}^{\theta}\right)-H\left(\alpha_{2}^{\theta}, q_{\theta}-\delta x_{2}^{\theta}\right)= & H\left(\alpha_{1}^{\theta}, q_{\theta}+\delta x_{1}^{\theta}\right)-H\left(\alpha_{2}^{\theta}, q_{\theta}+\delta x_{1}^{\theta}\right) \\
& +H\left(\alpha_{2}^{\theta}, q_{\theta}+\delta x_{1}^{\theta}\right)-H\left(\alpha_{2}^{\theta}, q_{\theta}-\delta x_{2}^{\theta}\right) \\
\leq & C\left(\left\|\alpha_{1}^{\theta}-\alpha_{2}^{\theta}\right\|_{B}\left(1+\left|q_{\theta}\right|+\delta\left(\left|x_{1}^{\theta}\right|+\left|x_{2}^{\theta}\right|\right)\right)\right) \\
& +C\left(\left(1+\left\|\alpha_{2}^{\theta}\right\|\right) \delta\left(\left|x_{1}^{\theta}\right|+\left|x_{2}^{\theta}\right|\right)\right) \\
= & o(\sqrt{\varepsilon}) o\left(\frac{1}{\sqrt{\varepsilon}}\right)+o\left(\frac{1}{\sqrt{\delta}}\right) o(\sqrt{\delta}) \rightarrow 0 \text { as } \theta \rightarrow\left(0^{+}, 0^{+}\right) .
\end{aligned}
$$

Putting everything together and passing to the limit in (5.15) then yields $\lambda M \leq 0$ so that the proof is complete.

We have already noticed that if $A$ is $H^{1}$ then $v$ defined by (2.5) actually belongs to $\mathrm{BUC}\left(\mathrm{E}_{\mathrm{B}}, \mathbb{R}\right)$. We thus deduce the following

Theorem 5.2. Assume that (H1), (H'2) and (H3) hold, then the value function $v$ defined by (2.5) is the only $\mathrm{BUC}\left(\mathrm{E}_{\mathrm{B}}, \mathbb{R}\right) \cap \mathrm{C}^{0}\left(\mathrm{E}_{\mathrm{w}}, \mathbb{R}\right)$ viscosity solution of $(4.6)$ on $\mathbb{R}^{d} \times L^{2}$.

\section{VARIANTS AND CONCLUDING REMARKS}

\subsection{Finite-dimensional reduction}

For the sake of simplicity, let us assume in this paragraph that $d=k=1$ and that $A$ is an exponential weight:

$$
A(s)=\mathrm{e}^{-\delta s}, \forall s>0 \text { with } \delta>0 .
$$

Defining the optimal control problem and its value function $v$ as in (2.5), it is easy to see that, under the special exponential form of $A, v$ actually depends on $z$ only through the scalar parameter $y(z):=\int_{0}^{\infty} \mathrm{e}^{-\delta s} z(s) \mathrm{d} s$. More precisely, setting:

$$
v(x, z)=w(x, y(z)), \forall(x, z) \in E \text { and } y(z):=\int_{0}^{+\infty} \mathrm{e}^{-\delta s} z(s) \mathrm{d} s
$$

it is easy to check that $v$ solves the infinite-dimensional Hamilton-Jacobi equation (4.6) if and only if $v$ is given by (6.2) and $w$ solves the two-dimensional equation:

$$
\lambda w(x, y)+H_{0}\left(x, y, \partial_{x} w(x, y)\right)-\partial_{y} w(x, y)(\delta y+x)=0,
$$

where

$$
H_{0}(x, y, p):=\sup _{u \in K}\{-L(x, u)-p \cdot F(x, u, y)\}, \forall(x, y, p) \in \mathbb{R}^{3} .
$$

This finite-dimensional reduction of the problem of course heavily relies on the exponential form (6.1). We refer to [20] for the extension of such finite-dimensional reduction in a stochastic setting. Finally, let us mention that the reduction to a finite-dimensional dynamics is also possible in the case where $A$ is constant and $z \in L^{1}$. 


\subsection{The evolutionary problem}

In the present article, we have focused on the stationary case. If we consider, the finite horizon optimal control problem

$$
v(t, x, z):=\inf _{u \in V}\left\{\int_{t}^{T} L\left(s, y_{t, x, z, u}(s), u(s)\right) \mathrm{d} s+g\left(y_{t, x, z, u}(T)\right)\right\}
$$

where $y_{t, x, z, u}$ denotes the solution of the Cauchy problem

$$
\begin{aligned}
& \dot{x}(s)=F\left(s, x(s), u(s), \int_{0}^{+\infty} A(\tau) x(s-\tau) \mathrm{d} \tau\right), t>0, \\
& \text { with initial conditions } x(t)=x, x(t-s)=z(s), s>0,
\end{aligned}
$$

this leads to the following evolution equation for $v$ :

$$
\partial_{t} v(t, x, z)+\inf _{u \in K}\left\{L(t, x, u)+\nabla_{x} v(t, x, z) \cdot \mathcal{F}(t, x, z, u)\right\}-\left\langle D_{z} v(t, x, z), \dot{z}\right\rangle=0,
$$

together with the boundary condition

$$
v(T, x, z)=g(x), \forall(x, z) \in E .
$$

Acknowledgements. The authors are grateful to an anonymous referee for his numerous and valuable suggestions that led to an improved presentation.

\section{REFERENCES}

[1] C.T.H. Baker, G.A. Bocharov and F.A. Rihan, A Report on the Use of Delay Differential Equations in Numerical Modelling in the Biosciences. Technical report, Manchester Centre for Computational Mathematics, UK (1999).

[2] A. Bensoussan, G. Da Prato, M. Delfour and S.K. Mitter, Representation and control of infinite dimensional systems. Second Edition, Birkhäuser (2007).

[3] M. Bardi and I.C. Dolcetta, Optimal Control and Viscosity Solutions of Hamilton-Jacobi-Bellman Equations. Birkhäuser, Boston (1997).

[4] G. Barles, Solutions de viscosité des équations de Hamilton-Jacobi, Mathematics and Applications 17. Springer-Verlag, Paris (1994).

[5] R. Boucekkine, O. Licandro, L. Puch and F. del Rio, Vintage capital and the dynamics of the AK model. J. Econ. Theory 120 (2005) 39-72.

[6] H. Brezis, Analyse fonctionnelle, théorie et applications. Masson, Paris (1983).

[7] G. Carlier and R. Tahraoui, On some optimal control problems governed by a state equation with memory. ESAIM: COCV 14 (2008) 725-743.

[8] M. Crandall and P.-L. Lions, Hamilton-Jacobi equations in infinite dimensions. I. Uniqueness of viscosity solutions. J. Funct. Anal. 62 (1985) 379-396.

[9] M. Crandall and P.-L. Lions, Hamilton-Jacobi equations in infinite dimensions. II. Existence of viscosity solutions. J. Funct. Anal. 65 (1986) 368-405.

[10] M. Crandall and P.-L. Lions, Hamilton-Jacobi equations in infinite dimensions. III. J. Funct. Anal. 68 (1986) $214-247$.

[11] M. Crandall and P.-L. Lions, Viscosity solutions of Hamilton-Jacobi equations in infinite dimensions. IV. Hamiltonians with unbounded linear terms. J. Funct. Anal. 90 (1990) 237-283.

[12] M. Crandall and P.-L. Lions, Viscosity solutions of Hamilton-Jacobi equations in infinite dimensions. V. Unbounded linear terms and B-continuous solutions. J. Funct. Anal. 97 (1991) 417-465.

[13] M. Crandall and P.-L. Lions, Hamilton-Jacobi equations in infinite dimensions. VI. Nonlinear $A$ and Tataru's method refined, in Evolution equations, control theory, and biomathematics, Lect. Notes Pure Appl. Math. 155, Dekker, New York (1994) 51-89.

[14] I. Elsanosi, B. Øksendal and A. Sulem, Some solvable stochastic control problems with delay. Stochast. Stochast. Rep. $\mathbf{7 1}$ (2000) 69-89. 
[15] G. Fabbri, Viscosity solutions to delay differential equations in demo-economy. Math. Popul. Stud. 15 (2008) $27-54$.

[16] G. Fabbri, S. Faggian and F. Gozzi, On dynamic programming in economic models governed by DDEs. Math. Popul. Stud. 15 (2008) 267-290.

[17] S. Faggian and F. Gozzi, On the dynamic programming approach for optimal control problems of PDE's with age structure. Math. Popul. Stud. 11 (2004) 233-270.

[18] F. Gozzi and C. Marinelli, Stochastic optimal control of delay equations arising in advertising models, in Stochastic partial differential equations and applications VII, Chapman \& Hall, Boca Raton, Lect. Notes Pure Appl. Math. 245 (2006) $133-148$.

[19] V.B. Kolmanovskii and L.E. Shaikhet, Control of systems with aftereffect, Translations of Mathematical Monographs. American Mathematical Society, Providence, USA (1996).

[20] B. Larssen and N.H. Risebro, When are HJB-equations in stochastic control of delay systems finite dimensional? Stochastic Anal. Appl. 21 (2003) 643-671.

[21] L. Samassi and R. Tahraoui, Comment établir des conditions nécessaires d'optimalité dans les problèmes de contrôle dont certains arguments sont déviés ? C. R. Math. Acad. Sci. Paris 338 (2004) 611-616.

[22] L. Samassi and R. Tahraoui, How to state necessary optimality conditions for control problems with deviating arguments? ESAIM: COCV 14 (2008) 381-409. 